\title{
Inklusionsbezogene Anforderungen in Zusammenhang mit personalen und beruflichen Ressourcen
}

\author{
Milena Peperkorn (D) Katharina Müller • Peter Paulus
}

Eingegangen: 30. Juli 2020 / Überarbeitet: 12. Mai 2021 / Angenommen: 21. Juni 2021 / Online publiziert: 12. November 2021

(C) Der/die Autor(en) 2021

Zusammenfassung Mit der Einführung inklusiver Schulen ändern sich berufliche Tätigkeiten von Lehrkräften. Bedingt durch heterogenere Lerngruppen arbeiten Lehrkräfte etwa öfter im multiprofessionellen Team, gestalten inklusive LehrLern-Prozesse oder diagnostizieren sonderpädagogische Förderbedarfe. Um mit den damit verbundenen Anforderungen umgehen zu können, benötigen Lehrkräfte Ressourcen. Erste Forschungsarbeiten im Kontext schulischer Inklusion stellen heraus, dass insbesondere personale Ressourcen wie eine positiv ausgeprägte Selbstwirksamkeitserwartung und berufliche Ressourcen wie die mitarbeiterinnen- und mitarbeiterorientierte Unterstützung der Schulleitung oder ein gemeinsames Schulethos, hilfreich beim Umgang mit inklusionsbezogenen Anforderungen sein könnten. Die vorliegende Studie, in der $N=6712$ Lehrkräfte schriftlich befragt wurden, untersucht unter Berücksichtigung der theoretischen Annahmen des Job Demands-Resources Modells anhand exemplarisch ausgewählter, inklusionsbezogener Tätigkeiten das Zusammenspiel zwischen Anforderungen, Ressourcen, Beanspruchungen und Engagement. Die Ergebnisse zeigen, dass alle Ressourcen positiv mit der Arbeitszufriedenheit korrelieren und die Selbstwirksamkeitserwartung darüber hinaus einen schwach abpuffernden Effekt auf die anforderungsbedingte Beanspruchung hat.

Dr. Milena Peperkorn $(\bowtie)$

Institut für Erziehungswissenschaft, Leibniz Universität Hannover, Schloßwender

Straße 1, 30159 Hannover, Deutschland

E-Mail: milena.peperkorn@iew.uni-hannover.de

Prof. Dr. Katharina Müller

Institut für Erziehungswissenschaft, Leibniz Universität Hannover, Schloßwender

Straße 1, 30159 Hannover, Deutschland

E-Mail: katharina.mueller@iew.uni-hannover.de

Prof. Dr. Peter Paulus

Zentrum für Angewandte Gesundheitswissenschaften (ZAG), Leuphana Universität Lüneburg, Wilschenbrucher Weg 84, 21335 Lüneburg, Deutschland

E-Mail: paulus@leuphana.de 
Schlüsselwörter Ressourcen · Inklusion · Lehrkräfte · Berufliche Anforderungen

\title{
Inclusion-related demands in relation to personal and occupational resources
}

\begin{abstract}
With the introduction of inclusive schools, the professional activities of teachers change. Due to more heterogeneous learning groups, teachers work more often in multi-professional teams, create inclusive teaching-learning processes and diagnose special educational needs. Teachers need resources in order to cope with the demands of inclusion. Initial research in the context of school inclusion shows that personal resources, such as self-efficacy, and professional resources, such as staff-oriented support from school management or a shared school ethos, could be particularly helpful in dealing with inclusion-related demands. The present study was conducted with $N=6712$ teachers. Taking into account the theoretical assumptions of the Job Demands-Resources Model, the study analyses the interaction between demands, resources, strain, and engagement in an initial approach using selected examples of inclusion-related activities. The results show that all resources correlate positively with job satisfaction and that, in addition, self-efficacy has a weak buffering effect on demand-induced strain.
\end{abstract}

Keywords Resources · Inclusion · Teacher · Job Demands

\section{Einleitung}

Mit der Umsetzung der Inklusionsreform werden schulische Lerngruppen heterogener (Klemm 2018). Dies führt zur Erweiterung der beruflichen Tätigkeiten von Lehrkräften: Sie sollen etwa Lehr-Lern-Settings gestalten, welche den individuellen Bedarfen aller Lernenden gerecht werden (Gräsel et al. 2017), sonderpädagogische Förderbedarfe diagnostizieren und im multiprofessionellen Team arbeiten (Schwab 2017). Damit kommt den Lehrkräften eine besondere Bedeutung für das Gelingen der Maßnahmen zu (Gräsel et al. 2017).

Es gibt Hinweise darauf, dass veränderte berufliche Tätigkeiten im Kontext von Reformen generell (Kunz Heim et al. 2014) und bezogen auf die Maßnahmen zur schulischen Inklusion im Besonderen (etwa Bosse et al. 2015; Ervasti et al. 2012) mit Beanspruchungen bei Lehrkräften in Zusammenhang stehen können. Weniger hingegen ist darüber bekannt, auf welche personalen und beruflichen Ressourcen Lehrkräfte beim Umgang mit inklusionsbezogenen Anforderungen zurückgreifen können.

Die vorliegende Arbeit nähert sich diesem Desiderat an und untersucht in Anlehnung an das Job Demands - Resources (JD-R) Modell (Bakker und Demerouti 2007) den Zusammenhang zwischen beruflichen Anforderungen, Ressourcen, Beanspruchung und Engagement. Im Vordergrund steht die Forschungsfrage, inwieweit personale und berufliche Ressourcen die potenzielle Beanspruchung durch inklusionsbezogene Anforderungen abschwächen können. Zur Beantwortung der Frage werden zunächst exemplarisch ausgewählte, inklusionsbezogene Tätigkeiten im Be- 
reich der Kooperation, der Diagnostik sowie der individuellen Förderung als berufliche Anforderungen modelliert und theoretisch angenommene Zusammenhänge mit der Beanspruchung geprüft. Ferner wird untersucht, inwieweit personale und berufliche Ressourcen den angenommenen Zusammenhang zwischen Anforderung und Beanspruchung abschwächen.

\section{Theoretischer Hintergrund zu den Folgen beruflicher Anforderungen}

Zur Analyse des komplexen Zusammenspiels von beruflichen Anforderungen, Ressourcen, Beanspruchungen und Engagement eignet sich das empirische geprüfte JD-R Modell von Bakker und Demerouti (2007). Das Modell geht von zwei Prozessen aus: Einer fokussiert die anforderungsbedingte Entstehung von Beanspruchungen (negative Folge), der andere die Entstehung von Engagement (positive Folge). Anforderungen sind als Gesamtheit aller Einflüsse aufzufassen, die auf eine Person wirken und gewisse „Anstrengungen zu ihrer Bewältigung erfordern“ (Cramer et al. 2018, S. 7). Speziell berufliche Anforderungen zeichnen sich durch zeitliche und inhaltliche Aspekte (Demerouti und Nachreiner 2019), wie etwa spezifische Tätigkeiten oder die Intensität bzw. Menge der Aufgaben aus (Rudow 1994).

Beanspruchungen als Resultat des Umgangs mit einer Anforderung sind mit Einbußen der Gesundheit bzw. des Wohlbefindens verbunden (Klusmann und Richter 2014). Zentrale Annahme des JD-R Modells ist, dass Ressourcen den Einfluss beruflicher Anforderungen auf Beanspruchungen ,abpuffern oder moderieren können“ (Demerouti und Nachreiner 2019, S. 124) und Beanspruchungen entsprechend geringer ausfallen, wenn ausreichend Ressourcen zum Umgang mit einer Anforderung zur Verfügung stehen. Ressourcen lassen sich dabei in interne, personale und externe, etwa berufsbezogene, Ressourcen voneinander unterscheiden (Becker 2006).

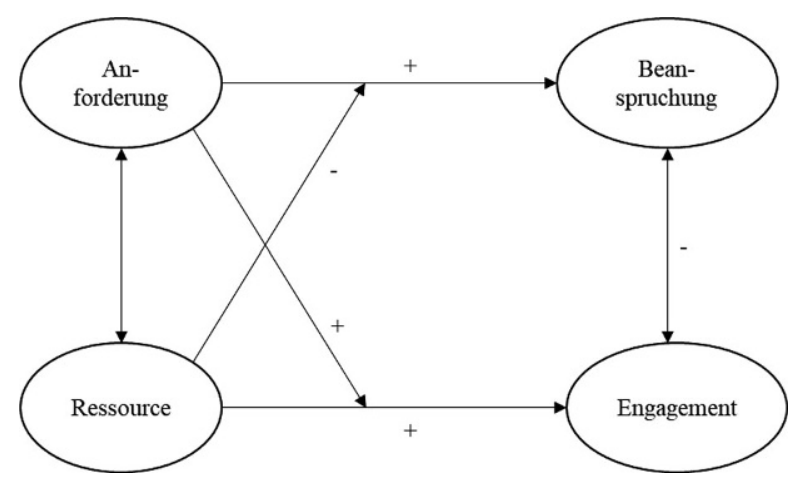

Abb. 1 Das Job Demands - Resources Model (adaptiert nach Bakker und Demerouti 2007). Berufliche Anforderungen stehen in Zusammenhang mit Beanspruchung. Ressourcen können die anforderungsbedingte Entstehung von Beanspruchung abpuffern. Ressourcen stehen in positivem Zusammenhang mit Engagement (bzw. assoziierten Konstrukten wie Arbeitszufriedenheit); werden Anforderungen positiv als „Challenge Stressoren“ eingeschätzt, können sie diesen positiven Zusammenhang fördern. Zwischen Beanspruchung und Engagement besteht ein negativer Zusammenhang 
Der erfolgreiche Umgang mit Anforderungen kann jedoch auch motivationales Potenzial (Turgut et al. 2014), wie die Erhöhung des Engagements, haben. Bakker und Demerouti (2017) konstatieren, dass das Modell den Einsatz unterschiedlicher Konstrukte ermöglicht, die in Verbindung mit Engagement stehen. So hat sich die Analyse von Arbeitszufriedenheit etabliert, da hier bedeutsame Zusammenhänge gezeigt wurden (Hulin und Judge 2003). Bei positiven Folgen können Anforderungen als „Challenge-Stressoren“ beschrieben werden (Demerouti und Nachreiner 2019). Zusätzlich zu den aufgeführten Prozessen adressiert das Modell die Beziehung zwischen Anforderungen und Ressourcen.

Das JD-R Modell ist nicht als inklusionsspezifisches Modell entwickelt worden. Der vorliegende Beitrag unternimmt den Versuch zu prüfen, ob sich anhand ausgewählter inklusionsbezogener Anforderungen die im Modell postulierten Zusammenhänge darstellen lassen. Im Folgenden werden die Teilkomponenten des JD-R Modells ausführlicher beschrieben und auf inklusionsbezogene Anforderungen enggeführt (Abb. 1).

\subsection{Anforderungen im Kontext inklusiver Bildung}

Mit dem Fokus auf inklusionsbezogenen Anforderungen des Lehrkräfteberufs, sei zu deren Einordnung zunächst eine Inklusionsdefinition vorangestellt. Inklusion wird für den Beitrag auf schulische Kontexte enggeführt und bezieht sich, im Sinne der „Zwei-Gruppen-Definition“ (Grosche 2015), auf die gemeinsame Beschulung von Schülerinnen und Schülern mit und ohne sonderpädagogische Förderbedarfe (SPF). Veränderte Lerngruppenzusammensetzungen gehen mit Anforderungen einher, die als entsprechende Tätigkeitsvorgaben für Lehrkräfte in den Rechts- und Verwaltungsvorschriften der Länder verankert sind (etwa Niedersächsisches Kultusministerium 2013). Die vorgenommene Engführung von Inklusion ermöglicht es, spezifische Tätigkeitsvorgaben des Lehrkräfteberufs genauer zu untersuchen. Einschränkend sei aber betont, dass diese Definition zur Vernachlässigung wichtiger Aspekte des Inklusionsdiskurses führt, wie etwa der Wertschätzung von Vielfalt oder die Vermeidung stigmatisierender Kategorisierungen (Göransson und Nilholm 2014).

Vor dem Hintergrund dieser Definition von Inklusion zählen zu den erweiterten Tätigkeiten für Lehrkräfte etwa die Bereiche Zusammenarbeit, Diagnostik und individuelle Förderung. Die Zusammenarbeit umfasst sowohl die „Kooperation“ von Regel- und Förderschullehrkräften, als auch die ,multiprofessionelle Zusammenarbeit“" von Lehrkräften und weiterem pädagogischen Personal, wie etwa Schulbegleiterinnen und -begleitern und wird als zentraler Gelingens- und Umsetzungsaspekt inklusiver Schulen betrachtet (Schwab 2017; Werning und Arndt 2013). Im Zuge der Umstrukturierung von Förderschulen und der damit einhergehenden Beschäftigung von Förderschullehrkräften an Regelschulen ist die Zusammenarbeit zudem auch elementarer Bestandteil von Schulentwicklungsprozessen.

Ein weiterer Bereich ist die Diagnostik. Die diagnostische Arbeit von Lehrkräften ist eine wichtige Voraussetzung für eine angemessene Vorbereitung, Durchführung und Nachbereitung von Unterricht (McElvany et al. 2009). Durch die Inklusionsreform sind Lehrkräfte nicht nur mit diagnostischen Verfahren zur Feststellung sonder- 
pädagogischer Förderbedarfe (Niedersächsisches Kultusministerium 2013) betraut, sondern auch mit unterschiedlichen Konzepten der (sonder-)pädagogischen Diagnostik konfrontiert, die sich in ihren Zielsetzungen, Einsatzbereichen und Umsetzungsformen z.T. deutlich unterscheiden (Neumann und Lütje-Klose 2020). Dies verlangt Lehrkräften in inklusiven Schulen nicht nur spezifische Kenntnisse ab (Sälzer et al. 2015), sondern kann sie bei der Durchführung vor neue Anforderungen stellen.

Schließlich ist als Anforderung der Bereich der individuellen Förderung bzw. die verstärkte Adaptation unterrichtlicher Angebote als Leitprinzip einer inklusiven Didaktik (Kullmann et al. 2014) zu nennen. Auch dieser Bereich ist nicht grundsätzlich neu für Lehrkräfte (Dumont 2019). Er zieht jedoch erweiterte Anforderungen in Hinblick auf eine veränderte Zusammensetzung der Schülerinnen- und Schülerschaft nach sich, wobei entsprechend auf die individuelle Förderung von mit Lernenden mit und ohne SPF rekurriert wird.

Im Kontext inklusiver Schulen ist davon auszugehen, dass mit der Erweiterung der Tätigkeiten auch deren Häufigkeit zunimmt. Entsprechend der theoretischen Annahmen zu Arbeitsanforderungen (Demerouti und Nachreiner 2019) kann angenommen werden, dass neben der Erweiterung der Tätigkeiten (inhaltliche Anforderung) auch deren häufigeres Vorkommen (zeitliche Anforderung) im Beruf mit einer Beanspruchung assoziiert ist. So weisen etwa Turgut et al. (2014) einen negativen Zusammenhang nach zwischen der psychischen Gesundheit von Lehrkräften und zusätzlichen beruflichen Verpflichtungen, einer erhöhten quantitativen Arbeitsbelastung sowie Zeitdruck. Gleichzeitig wurde gezeigt, dass eine Arbeitsintensivierung mit einer verringerten Arbeitszufriedenheit in Zusammenhang steht (Paškvan und Kubicek 2017).

Die aufgeführten Tätigkeiten sind beispielhaft zu verstehen und erheben nicht den Anspruch der Vollständigkeit der mit Inklusion einhergehenden Aufgaben für Lehrkräfte. Sie stellen vielmehr eine exemplarische Auswahl auf der Grundlage niedersächsischer Verwaltungsvorschriften für Lehrkräfte dar, um sich der empirischen Analyse inklusionsbezogener Tätigkeiten anzunähern. In systematischen Literaturreviews zählen die ausgewählten Tätigkeiten neben weiteren Anforderungen zu den zentralen „Aufgaben von Lehrkräften in inklusiven Bildungssystemen“ (Melzer et al. 2015, S. 63) und werden beschrieben ,als neue oder zumindest erweiterte Anforderungen, die Lehrerinnen und Lehrer beruflich meistern müssen“" (König et al. 2019, S. 43). Ergänzt werden könnten etwa Tätigkeiten in den Bereichen der Schulentwicklung (Weiß et al. 2019), der Beratung (z. B. Eltern) (König et al. 2019) oder der außerschulischen Kooperationen (Melzer et al. 2015).

\subsection{Folgen im Umgang mit beruflichen Anforderungen - Beanspruchung und Engagement}

Ein prominentes Konstrukt zur Ermittlung beruflicher Beanspruchung ist das Burnout-Syndrom (Maslach et al. 1997). Auf Grund von Abgrenzungsschwierigkeiten zum Konstrukt der Depressionen wird die Erfassung und Interpretation von Burnout jedoch auch kritisch diskutiert (Rothland und Klusmann 2016). Die Erhebung des Konstrukts Irritation stellt eine geeignete Alternative dar, da ebenfalls die berufli- 
che Beanspruchung in der Folge von Arbeitsanforderungen erfasst wird. Es kann hier zwischen einer emotionalen (Gereiztheit) und einer kognitiven Komponente (Rumination) der Irritation als Indikator tätigkeitsspezifischer Arbeitsanforderungen unterschieden werden (Mohr et al. 2005), wobei Letztere etwa die gedankliche Beschäftigung mit Arbeitsanforderungen nach Feierabend oder das Abschalten nach der Arbeit allgemein beinhaltet. Eine erhöhte Ausprägung der Irritation kann als Beeinträchtigung der psychischen Gesundheit interpretiert werden (Dadaczynski et al. 2019).

Erste Forschungsarbeiten haben sich mit der Beanspruchung bei der Arbeit in inklusiven Settings befasst. So zeigen Bosse et al. (2015), dass das selbstberichtete Beanspruchungserleben von Lehrkräften mit der Einführung inklusiver Schulen angestiegen ist. Ervasti et al. (2012) berichten auf der Grundlage von steigenden krankheitsbedingten Fehlzeiten an inklusiven Schulen einen Anstieg des Beanspruchungserlebens. Etwas spezifischer zeigen Hinds et al. (2015), dass insbesondere die Arbeit mit Schülerinnen und Schülern mit SPF mit einer erhöhten Beanspruchung der Lehrkräfte verbunden ist. Diesen Forschungsergebnissen gegenüber steht das Ergebnis der Studie von Weiß et al. (2019), die keine Zusammenhänge zwischen inklusionsbezogenen Anforderungen im Bereich der Schulentwicklung und Depressionen fanden.

Zur Erfassung positiver Folgen hat sich im Rahmen des JD-R Modells die Erfassung von Arbeitszufriedenheit etabliert (Turgut et al. 2014). Nach Klusmann (2011) ist Arbeitszufriedenheit das „Resultat eines erfolgreichen Umgangs mit den Belastungen des Berufs“ (Klusmann 2011, S. 280). Da Arbeitszufriedenheit in diesem Sinne auch kognitive und emotionale Bewertungen berücksichtigt (Weiss 2002), kann sie insgesamt als ,motivational bedeutsames Konstrukt angesehen werden“ (Schult et al. 2014, S. 61), welches eine Nähe zu den Konstrukten Wohlbefinden und Leistungsfähigkeit aufweist (Gehrmann 2013). Ressourcen wie etwa das Vorhandensein sozialer Unterstützungssysteme an der Einzelschule können die Arbeitszufriedenheit steigern (Turgut et al. 2014).

Empirische Studien zeigen, dass - trotz der Anforderungsintensität des Berufs die Arbeitszufriedenheit bei Lehrkräften insgesamt hoch ausgeprägt ist (Krause und Dorsemagen 2014). Schult et al. (2014) zufolge berichten Lehrkräfte beim Vergleich mit anderen sozialen Berufsgruppen im Mittel die numerisch höchste Arbeitszufriedenheit.

\subsection{Berufliche und personale Ressourcen}

Es wurden in vergangenen Jahren verstärkt Forschungsvorhaben durchgeführt, die weniger auf die Vermeidung oder Kompensation von Beanspruchungen als vielmehr auf die Stärkung von Ressourcen zielen (Döring-Seipel und Dauber 2010). Unter dieser Perspektive sind auch in Bezug auf die Einführung inklusiver Schulen Arbeiten entstanden (etwa Bennemann 2019; Kiel et al. 2016; Rathmann und Hurrelmann 2018), die bedeutsame berufliche und personale Ressourcen im Umgang mit inklusionsbezogenen Anforderungen herausarbeiten.

Berufliche Ressourcen beziehen sich zum einen auf die Organisation selbst und beinhalten die institutionellen Bedingungen zur Aufgabenerfüllung. Im schulischen 
Kontext sind dies bspw. geteilte gemeinsame Werte. Zum anderen können berufliche Ressourcen auf zwischenmenschliche Beziehungen referenzieren, wie etwa die Unterstützung durch eine Führungskraft (Demerouti und Nachreiner 2019).

Die Bedeutsamkeit gemeinsamer pädagogischer Vorstellungen (Schulethos) innerhalb eines Kollegiums betonen Rathmann und Hurrelmann (2018). Das Konstrukt des Schulethos kann auf Ebene des Kollegiums angesiedelt werden und bezieht sich z. B. auf schulische Regeln, den Umgang mit Lernenden oder einen Konsens bei der Schulphilosophie. Im Vergleich mit dem Konstrukt Schulklima (Wang und Degol 2016) finden sich Überschneidungen in Hinblick auf die Entwicklung und Etablierung gemeinsamer Ziele. Das Schulethos ist jedoch weniger umfassend angelegt und beinhaltet z. B. Partizipationsmöglichkeiten nicht. Vorrangig bedeutsam beim Schulethos ist nicht dessen Spezifik, sondern die einheitliche Ausrichtung innerhalb einer Einzelschule. Das Vorhandensein eines Schulethos wurde als Faktor mit hoher (positiver) Relevanz für die Arbeitszufriedenheit (Nübling et al. 2012) und das psychische Wohlbefinden identifiziert (Baeriswyl et al. 2017). Urton (2016) stellt die Bedeutsamkeit des konsistenten Handelns schulischer Akteure bei (inklusiven) Schulentwicklungsaufgaben im Hinblick auf das pädagogische Leitbild der Schule heraus. Darüber hinaus verdeutlichen Lütje-Klose et al. (2018), dass ein gemeinsam getragenes Schulethos auch bei der individuellen Förderung von Lernenden mit SPF förderlich ist.

Als weitere wichtige berufliche Ressource wird die Unterstützung durch die Schulleitung benannt (Krause und Dorsemagen 2014). Voraussetzung dafür ist ein positiver Führungsstil (Turgut et al. 2014), der sich etwa durch eine Mitarbeiterinnen- und Mitarbeiterorientierung auszeichnet. Je intensiver sich eine Führungskraft für die Interessen der Mitarbeitenden einsetzt, desto höher ist die Arbeitszufriedenheit ausgeprägt (Judge und Piccolo 2004). Die Bedeutsamkeit der Schulleitung zeigt sich auch bei inklusionsbezogenen Anforderungen. Bennemann (2019) führt auf, dass Lehrkräfte, die in inklusiven Settings arbeiten, die Schulleitung als bedeutsame Ressource betonen. Dabei wird neben der organisationsbezogenen auch die emotionale Unterstützung betont (Bennemann 2019). So kann die Schulleitung Entlastung durch das positive Beeinflussen des Sozialklimas bedingen (Urton et al. 2018).

Personale Ressourcen sind, auch in Abgrenzung zu den beruflichen Ressourcen, in der jeweiligen Person (kognitiv) repräsentiert (Cramer et al. 2018). Hierbei zeigt sich insbesondere die Selbstwirksamkeitserwartung (SWE) als bedeutsame Ressource für den Umgang mit Anforderungen (Rothland und Klusmann 2016). Bandura (1997) beschreibt SWE als subjektive Überzeugung eines Individuums, mit bestimmten Anforderungen erfolgreich umgehen zu können. Demnach führen positive Erwartungen bezüglich der eigenen Kompetenz zu einem verringerten Auftreten emotionaler Beanspruchung (Schmitz und Schwarzer 2000). Von der allgemeinen SWE lässt sich die domänenspezifische, etwa berufsbezogene SWE unterscheiden (Kunz Heim et al. 2019). Spezifisch für den Lehrkräfteberuf konnte gezeigt werden, dass eine positiv ausgeprägte SWE eine abschwächende Wirkung auf Beanspruchungen hat (etwa Schwarzer und Warner 2014). Im Kontext schulischer Inklusion werden SWE als zentral angenommen, da sich Personen mit einer positiver ausgeprägten SWE größeren Herausforderungen stellen und mehr Ausdauer bei der Erreichung 
von Zielen haben (Greiner et al. 2020). Lehrkräfte mit höher ausgeprägten SWE tragen dementsprechend stärker zur Umsetzung inklusiver Schulen bei (Kiel et al. 2019). Während einerseits ein Bedarf an selbstwirksamkeitsbezogener Forschung im Kontext der sonderpädagogischen Bildung auch mit Blick auf die gesundheitsbezogene Prävention konstatiert wird (Kiel et al. 2016), können Bosse et al. (2016) den prädiktiven Effekt der SWE auf das Beanspruchungserleben im inklusiven Setting nicht bestätigen.

\section{Methode}

\subsection{Fragestellung und Hypothesen}

Für Lehrkräfte erwachsen aus der Umsetzung inklusiver Schulen erweiterte berufliche Anforderungen, mit denen sie durch den Einsatz der ihnen zur Verfügung stehenden Ressourcen umgehen müssen. Abgeleitet von den Ausführungen des dargelegten theoretischen Hintergrunds untersucht der Beitrag den Zusammenhang zwischen der Häufigkeit des Vorkommens exemplarisch ausgewählter, inklusionsbezogener Anforderungen (Kooperation, Diagnostik, individuelle Förderung von Lernenden mit SPF) und der beruflichen Beanspruchung sowie den personalen und beruflichen Ressourcen. Bei den Ressourcen wurden insbesondere solche berücksichtigt, deren „linderndes“ bzw. ,abpufferndes“ Potenzial in Vorarbeiten herausgestellt wurde. Im Mittelpunkt der vorliegenden Arbeit steht die Frage: Welche personalen und beruflichen Ressourcen schwächen die potenzielle Beanspruchung durch inklusionsbezogene Anforderungen ab? Die Hypothesen, die im Anschluss an die Forschungsfrage geprüft werden, lauten:

H1 Ein mitarbeiterinnen- und mitarbeiterorientiertes Führungsverhalten der Schulleitung schwächt den Zusammenhang von inklusionsbezogenen Anforderungen und Beanspruchung bei Lehrkräften ab.

H2 Das Vorhandensein gemeinsamer pädagogischer Vorstellungen (Schulethos) innerhalb des schulischen Kollegiums schwächt den Zusammenhang von inklusionsbezogenen Anforderungen und Beanspruchung bei Lehrkräften ab.

H3 Eine positiv ausgeprägte, berufsbezogene Selbstwirksamkeitserwartung schwächt den Zusammenhang von inklusionsbezogenen Anforderungen und Beanspruchung bei Lehrkräften ab.

\subsection{Die Studie Mehr Zeit für gute Schule}

Die vorliegende Untersuchung ist Teil der Studie Mehr Zeit für gute Schule (Paulus et al. 2017) aus dem Jahr 2016. Ziel der in Niedersachsen durchgeführten Querschnittsstudie ist es, aussagekräftige Informationen über den Einfluss bildungspolitischer Reformen, wie etwa die Einführung inklusiver Schulen, sowie deren Umsetzung durch Rechts- und Verwaltungsvorschriften am Arbeitsplatz zu erlangen. 


\subsection{Stichprobe}

Die Stichprobe der vorliegenden Arbeit umfasst $N=6712$ Personen. 74,2\% der befragten Lehrkräfte geben an weiblich zu sein. Dies entspricht etwa der Quote in der niedersächsischen Grundgesamt der Regelschullehrkräfte (72,3\%) (alle Referenzwerte des Jahres 2016: Niedersächsisches Kultusministerium 2018). Das Durchschnittsalter liegt bei $M=43,2$ Jahren $(S D=10,4)$ (Referenz $M=44,3)$ und die Berufsjahre bei $M=14,7$ Jahren $(S D=10,1)$. Entsprechend der Verteilung der Grundgesamtheit haben Lehrkräfte von Grundschulen mit 39,7\% (Referenz 30,7\%) am häufigsten an der Befragung teilgenommen. Es folgen Lehrkräfte aus Gymnasien $(17,6 \%)$ (Referenz 25,1\%), Oberschulen (12,5\%) (Referenz 12,1\%), Integrierten Gesamtschulen (8,4\%) (Referenz 9,1\%), Realschulen (7,6\%) (Referenz 6,6\%), Förderschulen (6,7\%) (Referenz 7,3\%), Kooperativen Gesamtschulen (4,3\%) (Referenz 4,9\%) und Hauptschulen (3,2\%) (Referenz 4,2\%). Die Schulformzugehörigkeit wurde über die Frage erhoben, an welcher Schule die Lehrkräfte hauptsächlich unterrichten. Da in einem inklusiven Schulsystem, wie voranstehend herausgearbeitet, auch für Förderschullehrkräfte $(n=447)$ neue Anforderungen resultieren können, wurden Lehrkräfte die ,hauptsächlich“ an einer Förderschule unterrichten ebenfalls in die vorliegenden Analysen aufgenommen.

\subsection{Instrumente}

Zur Erfassung inklusionsbezogener Anforderungen wurden in einem diskursiven Prozess mit Vertreterinnen und Vertretern der niedersächsischen Schulverwaltung und Bildungspolitik, berufliche Tätigkeiten aus geltenden Rechts- und Verwaltungsvorschriften für schulisches Personal in Niedersachsen ausgewählt. Beispielhaft sei hier die ,Verordnung zur Feststellung eines Bedarfs an sonderpädagogischer Unterstützung“ (Niedersächsisches Kultusministerium 2013) genannt, auf Grundlage derer die Tätigkeit Diagnostik operationalisiert wurde. Vier Tätigkeiten bilden den Ausgangspunkt der vorliegenden Analyse (Tab. 1). Sie rekurrieren auf die aufgeführten, inklusionsbezogenen Tätigkeitsbereiche schulischen Personals (Kooperation, Diagnostik, individuelle Förderung von Lernenden mit SPF). Jede der Tätigkeiten (inhaltliche Anforderung) wurde in Hinblick auf deren Vorkommen im Arbeitsalltag (zeitliche Anforderung) auf einer vierstufigen Likert-Skala bewertet. Mit der Berücksichtigung von unterschiedlichen sowie verschieden häufig vorkommenden Tätigkeiten (Demerouti und Nachreiner 2019) soll die breite Erfassung von Anforderungen gewährleistet werden. Konkret schätzten die Lehrkräfte bspw. die Tätigkeit „Diagnostik“ („Die Durchführung des Verfahrens zur Feststellung des sonderpädagogischen Unterstützungsbedarfs im schulischen Bereich ist eine Tätigkeit, die in meinem Arbeitsalltag oft vorkommt“) in den Antwortoptionen ,,trifft zu“, ,trifft eher zu“, „trifft eher nicht zu“ und „trifft nicht zu“ ein.

Als berufliche Ressource wurde zum einen das „Mitarbeiterorientierte Führungsverhalten der Schulleitung“ (Schulleitung) anhand der Skala von Krause (2004) erhoben. Die Skala erfasst mit vier Items die wahrgenommene Unterstützung der Schulleitung aus Sicht schulischer Akteure. Zum anderen wurden „Gemeinsame pädagogische Vorstellungen“ innerhalb der Schule (Schulethos) (Krause 2004) mit 
Tab. 1 Inklusionsbezogene Anforderungen (Itemstämme)

\begin{tabular}{|c|c|}
\hline \multirow[t]{2}{*}{ Kooperation } & $\begin{array}{l}\text { „Die Zusammenarbeit der Regelschullehrkräfte und Förderschullehrkräfte für die Planung } \\
\text { und Durchführung eines gemeinsamen Unterrichts ist eine Tätigkeit ...“ }\end{array}$ \\
\hline & $\begin{array}{l}\text { „Die Zusammenarbeit der Lehrkräfte und der pädagogischen Mitarbeiterinnen und Mitar- } \\
\text { beiter in unterrichtsbegleitender Funktion für die Planung und Durchführung des Unter- } \\
\text { richts ist eine Tätigkeit ...“ }\end{array}$ \\
\hline Diagnostik & $\begin{array}{l}\text { „Die Durchführung des Verfahrens zur Feststellung des sonderpädagogischen Unterstüt- } \\
\text { zungsbedarfs im schulischen Bereich ist eine Tätigkeit ...“ }\end{array}$ \\
\hline Förderung & $\begin{array}{l}\text { „Die individuelle Förderung von Schülerinnen und Schülern mit unterschiedlichem son- } \\
\text { derpädagogischen Unterstützungsbedarf im Unterricht ist eine Tätigkeit ...“ }\end{array}$ \\
\hline
\end{tabular}

Tätigkeiten basierend auf niedersächsischen Rechts- und Verwaltungsvorschriften; Beantwortung des Items durch die Satzergänzung ,die in meinem Arbeitsalltag häufig vorkommt“ auf einer vierstufigen Likert-Skala

fünf Items erfasst, die nach gemeinsamen pädagogischen Prozess- und Zielvorstellungen schulischen Personals fragen.

Als personale Ressource wurden die beruflichen SWE der Lehrkräfte mit sechs Items der Skala „Lehrer-Selbstwirksamkeit“ von Schwarzer und Jerusalem (1999) erhoben. Im Rahmen der Faktorenanalyse wurde die Skala von zehn auf sechs Items gekürzt. Items zum Umgang mit Schülerinnen und Schülern, zur Unterrichtsgestaltung sowie zur Kooperation mit Kolleginnen und Kollegen sind - entsprechend der ausgewählten Tätigkeiten - weiterhin enthalten.

Die Beanspruchung wurde über den Indikator „Irritation“ anhand der gleichnamigen Skala von Mohr et al. (2005) erfasst. Für die vorliegende Analyse wurde die Teilskala zur berufsbezogenen Rumination genutzt. Mit dem Einsatz der Teilskala soll der Fokus auf explizit berufsbezogene Anforderungen und Beanspruchungen möglichst stringent verfolgt werden. Die Skala „Irritation“ und die zuvor aufgeführte Skala „Schulleitung“ beinhalten je ein Item, dessen eher gering ausgeprägte Faktorladung (s. Abb. 2) auf das Optimierungspotenzial der empirischen Erfassung der theoretischen Konstrukte hinweisen. Der Ausschluss dieser Items ergab für die vorliegende Analyse jedoch keine grundlegende Verbesserung der Fitstatistik der geprüften Modelle, weshalb sich für den Erhalt der Items in den Analysen entschieden wurde.

Als positive Folge wurde die „Arbeitszufriedenheit“ der Lehrkräfte anhand einer Ein-Item-Skala erfasst (Nübling et al. 2012). Obwohl Multi-Item-Skalen generell eine höhere Reliabilität aufweisen, ist die Erfassung der Arbeitszufriedenheit mittels Ein-Item-Skala zufriedenstellend (Judge et al. 2002).

Beispielitems aller verwendeter Skalen, deren deskriptive Statistik und die Angaben zur internen Konsistenz sind Tab. 2, eine vollständige Auflistung der Items sind dem Onlinematerial 1 zu entnehmen. Die interne Konsistenz der Skalen „Häufigkeit der Tätigkeitsausführung“ $(\alpha=0,76)$ und „Lehrer-Selbstwirksamkeit“ $(\alpha=0,70)$ ist als akzeptabel, die der übrigen Skalen als gut zu beurteilen (Bühner 2011). Hieraus mögliche resultierende Schwierigkeiten werden in der Diskussion aufgegriffen. 
Tab. 2 Beispielitems, Reliabilität, Itemanzahl und deskriptive Statistik einbezogener Skalen und Variablen

\begin{tabular}{|c|c|c|c|c|c|c|}
\hline Skala & Beispielitem & $\alpha$ & $\begin{array}{l}\text { Item- } \\
\text { anzahl }\end{array}$ & $N$ & $M$ & $S D$ \\
\hline \multicolumn{7}{|c|}{ Inklusionsbezogene Anforderungen } \\
\hline $\begin{array}{l}\text { Häufigkeit der } \\
\text { Tätigkeitsaus- } \\
\text { führung } \\
\text { (Paulus et al. } \\
\text { 2017) } \\
\text { (4-stufig) }\end{array}$ & $\begin{array}{l}\text { „Die Zusammenarbeit der Regelschul- } \\
\text { lehrkräfte und der Förderschullehr- } \\
\text { kräfte für die Planung und Durchfüh- } \\
\text { rung eines gemeinsamen Unterrichts } \\
\text { ist eine Tätigkeit, die in meinem Ar- } \\
\text { beitsalltag oft vorkommt“" }\end{array}$ & 0,76 & 4 & 6519 & 2,76 & 0,91 \\
\hline \multicolumn{7}{|c|}{ Berufliche Ressourcen } \\
\hline $\begin{array}{l}\text { Schulleitung } \\
\text { (Kaempf und } \\
\text { Krause 2004) } \\
\text { (4-stufig) }\end{array}$ & $\begin{array}{l}\text { „Man erfährt durch die Schulleitung } \\
\text { ausreichend Unterstützung“ }\end{array}$ & 0,92 & 4 & 5939 & 2,91 & 0,82 \\
\hline $\begin{array}{l}\text { Schulethos } \\
\text { (Kaempf und } \\
\text { Krause 2004) } \\
\text { (4-stufig) }\end{array}$ & $\begin{array}{l}\text { „In Erziehungs- und Disziplinfragen } \\
\text { ziehen alle Kolleginnen und Kollegen } \\
\text { an einem Strang“ }\end{array}$ & 0,92 & 5 & 5949 & 3,37 & 0,86 \\
\hline \multicolumn{7}{|c|}{ Personale Ressourcen } \\
\hline $\begin{array}{l}\text { Lehrer-Selbst- } \\
\text { wirksamkeit } \\
\text { (Schwarzer } \\
\text { und Jerusalem } \\
\text { 1999) } \\
\text { (4-stufig) }\end{array}$ & $\begin{array}{l}\text { „Ich bin mir sicher, dass ich mich in } \\
\text { Zukunft auf individuelle Probleme der } \\
\text { Schülerinnen und Schüler noch besser } \\
\text { einstellen kann“ }\end{array}$ & 0,70 & 6 & 5839 & 2,82 & 0,67 \\
\hline \multicolumn{7}{|c|}{ Beanspruchung und Engagement } \\
\hline $\begin{array}{l}\text { Irritation } \\
\text { (Mohr et al. } \\
\text { 2005) } \\
\text { (7-stufig) }\end{array}$ & $\begin{array}{l}\text { „Ich muss auch nach Feierabend } \\
\text { an Schwierigkeiten bei der Arbeit } \\
\text { denken“ }\end{array}$ & 0,84 & 4 & 5902 & 4,71 & 1,34 \\
\hline $\begin{array}{l}\text { Arbeits- } \\
\text { zufriedenheit } \\
\text { (Nübling et al. } \\
\text { 2012) } \\
\text { (4-stufig) }\end{array}$ & $\begin{array}{l}\text { „Wenn Sie Ihre Arbeitssituation be- } \\
\text { trachten, wie zufrieden sind Sie mit } \\
\text { Ihrer Arbeit insgesamt, unter Berück- } \\
\text { sichtigung aller Umstände?“" }\end{array}$ & - & 1 & 5916 & 2,53 & 0,68 \\
\hline
\end{tabular}

Schulethos Gemeinsame pädagogische Vorstellungen, $\alpha$ Cronbachs alpha (entsprechend der Stichprobe); weitere Items der Skalen „Schulleitung“ und „Schulethos“ siehe Onlinematerial I

\subsection{Analysen}

Deskriptive Statistiken wurden in SPSS (Version 26), die Zusammenhangsanalysen in Mplus (Version 8) berechnet. Die Zusammenhänge wurden in Form von Strukturgleichungsmodellen geprüft. Fehlende Werte wurden durch die Verwendung der in Mplus implementierten robusten Maximum Likelihood (MLR) -Schätzung (Muthén und Muthén 1998-2017) berücksichtigt. Die Strukturgleichungsmodelle wurden in Anlehnung an das JD-R Modell formuliert. Als Anforderung fungiert jeweils die Skala „Häufigkeit der Tätigkeitsausübung“, als Beanspruchung die Skala „Irritation“ und als Engagement die Single-Item-Skala „Arbeitszufriedenheit“. Die Ressourcen (Schulleitung, Schulethos, SWE) wurden entsprechend der aufgestellten Hypothesen 
eingesetzt. Zur empirischen Prüfung der angenommenen Modelle wurden zunächst konfirmatorische Faktorenanalysen durchgeführt, wobei das Modell für jede Ressource betrachtet wurde. Die Ergebnisse zeigen, dass sich das theoretisch angenommene Modell in den empirischen Daten abbildet. Für die Ressource „Schulleitung“ $(d f=63, C F I=0,97, R M S E A=0,05, S R M R=0,07)$ kann die Fitstatistik als akzeptabel bewertet werden (Hu und Bentler 1999; Weiber und Mühlhaus 2010). Auf die Angabe des Chi-Quadrat-Test wurde aufgrund von dessen Sensitivität bei großen Stichproben verzichtet. Der Modellfit nimmt bei der Modellierung mit der Ressource "Schulethos“ $(d f=75, C F I=0,93, R M S E A=0,08, S R M R=0,06)$ sowie bei der Ressource „Selbstwirksamkeit“ $(d f=88, C F I=0,92, R M S E A=0,06, S R M R=0,07)$ ab. Hier liegen die Index-Werte des CFI leicht unterhalb der etablierten Cut-OffWerte (Hu und Bentler 1999), weisen jedoch darauf hin, dass das angenommene Modell besser zur Datenstruktur passt als das Nullmodell. Da die Ausprägung von RMSEA und SRMR als akzeptabel bewertet werden können (Weiber und Mühlhaus 2010), kann der Modellfit insgesamt als noch zu akzeptieren eingeordnet werden.

\section{Ergebnisse}

Die deskriptiven Statistiken der berücksichtigten Konstrukte sind in Tab. 2 aufgeführt. Die Lehrkräfte geben an, dass die Anforderungen, entsprechend der Itemformulierung in den Antwortoptionen, ,eher“ häufig im Arbeitsalltag vorkommen $(M=2,76, S D=0,91)$. Mittelwerte und Standardabweichungen der Ressourcen befinden sich jeweils auf einem mittleren, leicht positiven Niveau. Hervorzuheben ist hier die Skala Schulethos, die eine hohe Ausprägung aufweist $(M=3,37, S D=0,86)$. Irritation als Indikator der Beanspruchung hat einen leicht erhöhten Mittelwert $(M=4,71, S D=1,34)$, während die Arbeitszufriedenheit als Indikator des Engagements $(M=2,53, S D=0,68)$ weniger stark ausgeprägt ist.

Bei allen Strukturgleichungsmodellen ist der Zusammenhang zwischen Anforderung und Beanspruchung nur schwach ausgeprägt $(0,6<\beta<0,7, p<0,01)$. Es besteht erwartungskonform ebenfalls in allen Modellen ein negativer Zusammenhang zwischen Irritation und der Arbeitszufriedenheit $(-0,18<\beta<-0,22, p<0,01)$. Gemein ist den Modellen zudem, dass die inklusionsbezogenen Anforderungen keinen negativen

Abb. 2 Strukturgleichungsmodell mit der berufsbezogenen Ressource „Schulleitung“

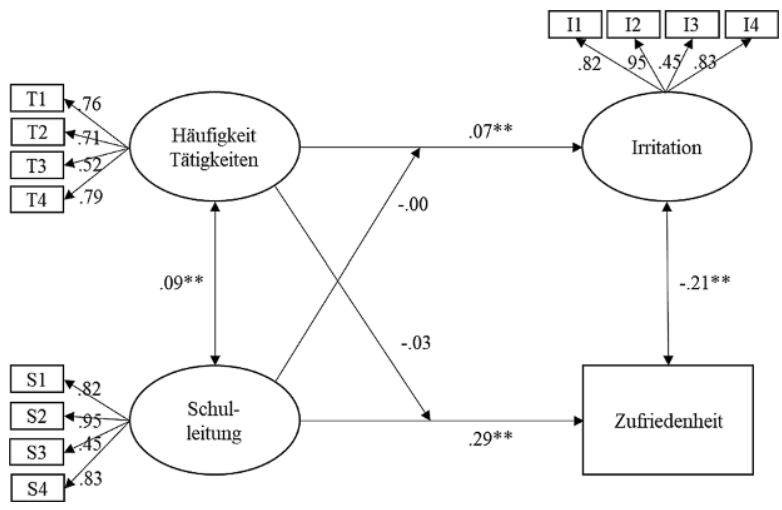


Abb. 3 Strukturgleichungsmodell mit der berufsbezogenen Ressource „Schulethos“

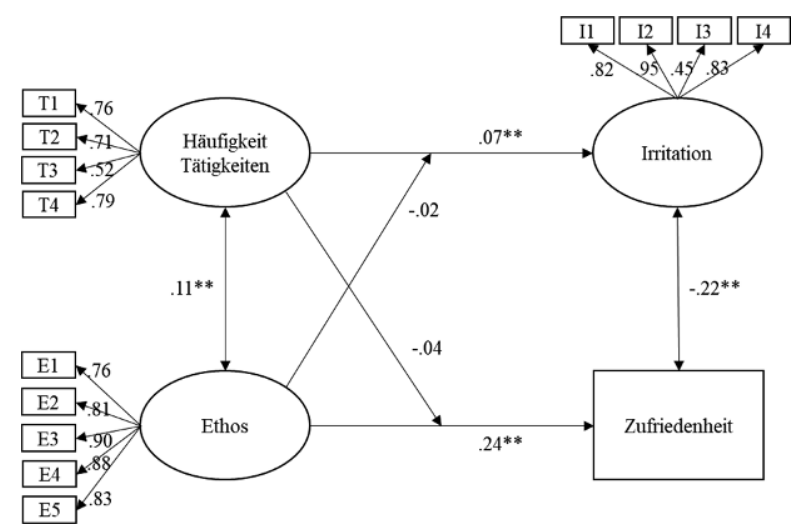

Einfluss auf den positiven Zusammenhang zwischen Ressource und Arbeitszufriedenheit haben. Schließlich besteht zwischen den Anforderungen und den geprüften Ressourcen ein signifikant positiver Zusammenhang. Alle Ressourcen-Pfade werden nachstehend, entsprechend der Hypothesen, aufgeführt.

Schulleitung Die Ressource Schulleitung korreliert positiv mit der Arbeitszufriedenheit $(\beta=0,29, p<0,01)$ : je positiver das Führungsverhalten der Schulleitung eingeschätzt wird, desto höher ausgeprägt ist auch die Arbeitszufriedenheit der Lehrkräfte. Gleichzeitig besteht ein positiver, wenn auch sehr geringer Zusammenhang zwischen der Ressource Schulleitung und den Anforderungen $(\beta=0,09, p<0,01)$. Für die Ressource Schulleitung lässt sich kein abschwächender Effekt auf die anforderungsbedingte Irritation feststellen. Die Hypothese (H1) muss dementsprechend verworfen werden (Abb. 2).

Schulethos Modellkonform steigt die Arbeitszufriedenheit signifikant an, je stärker die Lehrkräfte gemeinsame pädagogischen Vorstellungen im Kollegium wahrnehmen $(\beta=0,24, p<0,01)$. Die Korrelation mit den inklusionsbezogenen Anforderungen ist gering positiv ausgeprägt $(\beta=0,11, p<0,01)$, was darauf hindeutet, dass deren häufiges Vorkommen mit einem stärker ausgeprägten Schulethos zusammenhängt. Es zeigt sich kein Puffer-Effekt des Schulethos auf den Zusammenhang zwischen der Anforderung und der Beanspruchung. Die Hypothese (H2) muss daher verworfen werden (Abb. 3).

Selbstwirksamkeit Es besteht ein signifikant positiver Zusammenhang zwischen der beruflichen SWE und der Arbeitszufriedenheit $(\beta=0,35, p<0,01)$. Lehrkräfte mit stärker ausgeprägten SWE berichten dementsprechend eine höhere Arbeitszufriedenheit. Auch hier zeigt sich eine positive Korrelation zwischen der Selbstwirksamkeit und den inklusionsbezogenen Anforderungen $(\beta=0,10, p<0,01)$ : Häufigeres Vorkommen der inklusionsbezogenen Anforderungen steht in Zusammenhang mit höher ausgeprägten SWE. Bei der Ressource SWE zeigt sich der angenommene abschwächende Effekt bei der anforderungsbedingten Entstehung von Irritation. 
Abb. 4 Strukturgleichungsmodell mit der personalen Ressource ,Selbstwirksamkeit“

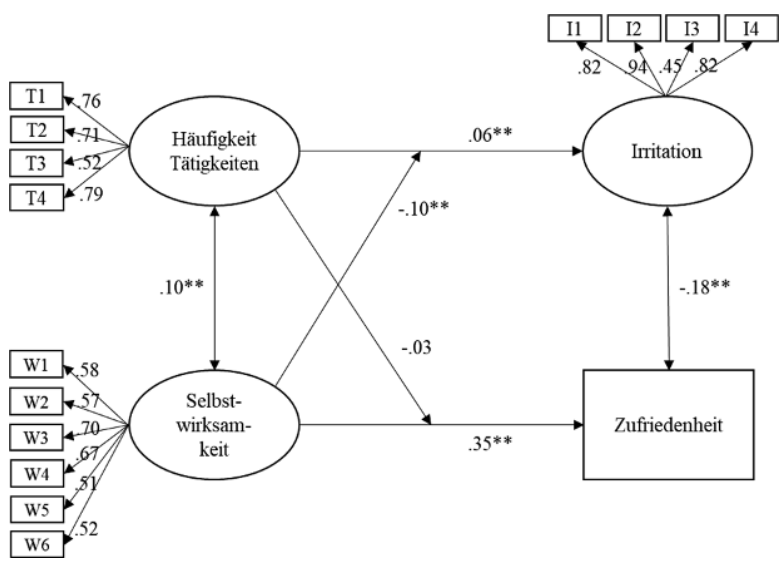

Dieser Effekt ist signifikant, aber von geringer Größe $(\beta=0,10, p<0,01)$. Die Hypothese (H3) wird folglich empirisch unterstützt (Abb. 4).

\section{Diskussion}

Der vorliegende Beitrag analysiert, welche personalen und beruflichen Ressourcen das Potenzial haben, eine mögliche Beanspruchung (Irritation) abzuschwächen, die durch inklusionsbezogene Anforderungen (Häufigkeit des Vorkommens inklusionsbezogener Tätigkeiten) für Lehrkräfte entstehen können. Unter Berücksichtigung der Annahmen des JD-R Modells (Bakker und Demerouti 2007) wurden Zusammenhänge mithilfe von Strukturgleichungsmodellen in den Blick genommen. Die Ergebnisse weisen darauf hin, dass das JD-R Modell auch für die Untersuchung inklusionsspezifischer Anforderungen geeignet ist. Drei Hypothesen wurden in Anschluss an die Forschungsfrage geprüft und werden nachstehend diskutiert.

$\mathrm{Zu}(\mathrm{H1})$ Ein mitarbeiterinnen- und mitarbeiterorientiertes Führungsverhalten der Schulleitung schwächt den Zusammenhang von inklusionsbezogenen Anforderungen und Beanspruchung bei Lehrkräften ab. Die Hypothese, die in Bezug auf die explorativen Ergebnisse von Bennemann (2019) sowie die Annahmen von Rathmann und Hurrelmann (2018) formuliert wurde, wird von den vorliegenden Ergebnissen nicht unterstützt. Aus der Analyse ist entsprechend abzuleiten, dass die Unterstützung der Lehrkräfte durch die Schulleitung nicht dazu beiträgt, dass Lehrkräfte mit den inklusionsbezogenen Anforderungen besser umgehen können. Mit der Arbeitszufriedenheit hingegen hängt das Führungsverhalten der Schulleitung signifikant positiv zusammen. Dieses Ergebnis steht in Einklang mit der Arbeit von Turgut et al. (2014) die zeigen konnten, dass soziale Unterstützungssysteme an der Einzelschule die Arbeitszufriedenheit steigern können.

$\mathrm{Zu}$ (H2) Das Vorhandensein gemeinsamer pädagogischer Vorstellungen innerhalb des schulischen Kollegiums (Schulethos) schwächt den Zusammenhang von inklusionsbezogenen Anforderungen und Beanspruchung bei Lehrkräften ab. Der Analyse nach stellt das Vorhandensein eines Schulethos im Kollegium keine Ressource dar, 
die die Lehrkräfte beim Umgang mit den ausgewählten Tätigkeiten in dem Maße unterstützt, dass die Entstehung von Beanspruchung gelindert wird. Die Annahme von Rathmann und Hurrelmann (2018) kann somit nicht bestätigt und H2 muss verworfen werfen. Gleichwohl zeigt sich, dass die Arbeitszufriedenheit steigt, je höher die Ressource Schulethos von den Lehrkräften wahrgenommen wird, was im Einklang mit Ergebnissen von Nübling et al. (2012) steht. Wie sich der Zusammenhang zwischen einem Schulethos und anderen Tätigkeiten (z.B. der Schulentwicklung; Urton 2016) darstellt, kann auf der Grundlage der vorliegenden Daten nicht geklärt werden.

(H3) Eine positiv ausgeprägte, berufliche Selbstwirksamkeitserwartung (SWE) schwächt den Zusammenhang von inklusionsbezogenen Anforderungen und Beanspruchung bei Lehrkräften ab. Die Hypothese wird durch die vorliegende Datenanalyse gestützt. Die berufliche SWE der Lehrkräfte puffert im geprüften Modell die anforderungsinduzierte Beanspruchung signifikant ab. Folglich ist der Zusammenhang von inklusionsbezogenen Anforderungen und der Beanspruchung bei Lehrkräften, die von der eigenen Wirksamkeit im Beruf überzeugt sind geringer ausgeprägt. Damit werden Forschungsarbeiten gestützt, die die Relevanz der SWE allgemein, und spezifisch auf inklusive Schulkontexte bezogen, herausarbeiten (Kiel et al. 2019). Die vorliegenden Ergebnisse unterstreichen den Bedarf an Gelegenheiten, im Rahmen derer Lehrkräfte an der Entwicklung einer positiven SWE arbeiten können. Denkbar wären hier etwa Lerngelegenheiten in denen (angehende) Lehrkräfte in Kontakt mit spezifischen, inklusionsbezogenen Anforderungen kommen, sie (begleitet) bearbeiten und sich in diesen Rahmen als wirksam erleben können z.B. in Anlehnung an das von Kunz Heim et al. (2019) evaluierte Training für Lehrkräfte, welches für inklusionsbezogene Anforderungen entsprechend adaptiert werden müsste.

Die Forschungsfrage lässt sich vor dem Hintergrund der aufgeführten Ergebnisse dahingehend beantworten, dass von den einbezogenen Ressourcen nur die personale Ressource SWE, nicht aber die beruflichen Ressourcen einen Einfluss auf die Beanspruchung hat. Gleichzeitig ist die Ausprägung des Puffer-Effekts niedrig ausgeprägt, was die Frage nach weiteren relevanten Ressourcen von Lehrkräften aufwirft, die im Rahmen künftiger Forschungsarbeiten geprüft werden sollten. Professionsspezifische Kompetenzen etwa im Bereich der Klassenführung als zentrale, auch belastungsrelevante Ressource (etwa Piwowar 2013) oder spezifische Weiterbildungsangebote seien hier beispielhaft aufgeführt.

Die Verbindung von Anforderung und Beanspruchung, lässt sich - auch modellbzw. theoriekonform (Bakker und Demerouti 2007) - empirisch zeigen. Es sei an dieser Stelle jedoch auf die geringen Zusammenhänge zwischen den inklusionsbezogenen Anforderungen und der Beanspruchung in den drei Modellen hingewiesen. Die geringe Ausprägung könnte in der Erfassung der Anforderung begründet sein. So wurden Aspekte schulischer Rahmenbedingungen nicht berücksichtigt, die eine vertiefte Interpretation der Anforderungen ermöglichen würden. Zu nennen sind hier etwa Kompositionsunterschiede innerhalb der Lerngruppen. Künftige Untersuchungen sollten dies zur besseren Interpretierbarkeit der Ergebnisse bedenken. Deutlich wird auch, dass bei den Anforderungen nicht von „Challenge Stressoren“ (Demerouti und Nachreiner 2019), etwa im Sinne selbst gewählter, inklusionsbezogener 
Aktivitäten oder Settings der Lehrkräfte, ausgegangen werden kann, da die Anforderungen die Zusammenhänge zwischen den Ressourcen und der Arbeitszufriedenheit nicht beeinflussen. Insgesamt ist bei diesem Aspekt mit Rekurs auf Weiß et al. (2019) darauf hinzuweisen, dass der Diskurs zu angenommenen Mehrbelastungen differenziert geführt werden muss.

$\mathrm{Zu}$ berücksichtigen ist weiter die eher geringe Reliabilität der Skala „Häufigkeit der Tätigkeitsausführung“. Da die Ausprägung der Reliabilität auch mit der Stärke der Korrelationen zusammenhängt (Danner 2015), können ungenaue Ableitungen die Folge sein. Eine vertiefte Operationalisierung der Tätigkeiten, z. B. anhand der Erfragung spezifischer Aspekte der Zusammenarbeit, Diagnostik oder individuellen Förderung könnten hier der Verbesserung der Reliabilität zuträglich sein. Auch aus der empirischen Erfassung der Beanspruchung könnte sich eine Einschränkung bei der Interpretation der Ergebnisse ergeben. Die Faktorladung eines Items der Skala „Irritation“ (Mohr et al. 2005) (H1-H3) ist eher gering ausgeprägt, was auf einen Überarbeitungsbedarf der empirischen Erfassung hindeutet. Selbige Einschränkung gilt für die Skala „Schulleitung“ (Krause 2004) (H2). Auch für künftige Untersuchungen ist dies kritisch zu berücksichtigen.

Die Vertiefung bzw. Ausweitung der zu erfassenden Tätigkeiten betrifft darüber hinaus auch den Einschluss der Förderschullehrkräfte in die vorliegenden Analysen. Kritisch zu diskutieren ist hier, ob Förderschullehrkräfte in gleichem Maße von den Anforderungen betroffen sind, wenngleich die Einführung inklusiver Schulen alle Lehrkräfte betrifft. Erste Analysen zu möglichen Gruppenunterschieden bei der Einschätzung inklusionsbezogener Anforderungen zwischen Regel- und Förderschullehrkräften wurden durch Vergleiche der latenten Mittelwerte geprüft und ergaben gering ausgeprägte, signifikante Unterschiede zugunsten der Förderschullehrkräfte etwa in Hinblick auf die „Bewältigbarkeit“ der Anforderungen (Peperkorn et al. 2020). Umfassende Analysen möglicher Gruppenunterschiede, auch in Hinblick auf weitere Anforderungen wie etwa den Bereich der Schulentwicklung (Weiß et al. 2019), stehen nach Kenntnisstand der Autor*innen jedoch zum jetzigen Zeitpunkt noch aus. Die Notwendigkeit einer Trennung in Regel- und Förderschullehrkräfte ist entsprechend für weitere Analysen zu prüfen.

Es ist zudem limitierend darauf hinzuweisen, dass in der vorliegenden Studie ein kausaler Effekt der Anforderungen zur Beanspruchung angenommen und modelliert wurde. Die Richtung des Zusammenhangs kann jedoch aufgrund des Querschnittdesigns nicht bestimmt werden.

Weitere Einschränkungen sind in einer möglichen Verzerrung durch Selbstselektion in der Stichprobe - bedingt durch die Freiwilligkeit an der Studienteilnahme zu sehen. Zudem stellen gesundheitlich beanspruchte Personen generell eine schwer erreichbare Zielgruppe (Healthy Worker Effect) dar. Auch Spillover-Effekte anderer Beanspruchungsquellen, die die Einschätzungen der befragten Lehrkräfte möglicherweise beeinflusst haben, können in den Analysen nicht berücksichtigt werden.

Abschließend bleibt festzuhalten, dass der zentrale Stellenwert der professionsspezifischen SWE im Umgang mit den hier untersuchten exemplarischen Anforderungen im Kontext inklusiver Schulen darauf schließen lässt, dass derart umfängliche Reformmaßnahmen mit substantiellen Unterstützungsmechanismen flankiert werden sollten. Die Bereitstellung externer Ressourcen und die Verbesserung der 
institutionellen Bedingungen spielen dabei eine wichtige Rolle für die Arbeitszufriedenheit der Lehrkräfte. Um jedoch die Entstehung von Beanspruchungen abpuffern zu können, bedarf es auch der systematischen Stützung personaler Ressourcen. Mit Blick auf die wesentlichen Quellen für die Entstehung der SWE, insbesondere die eigenen und stellvertretenden Erfahrungen (Bandura 1997) und deren kognitive Verarbeitung (Tschannen-Moran et al. 1998), rückt dies zum einen den Bereich der Fort- und Weiterbildungsmaßnahmen für Lehrkräfte, zum anderen den Bereich der Ausbildung angehender Lehrkräfte in den Blick. Aufgrund der sich im Laufe des Berufslebens zunehmend verfestigenden SWE (Tschannen-Moran et al. 1998) kann der frühzeitigen Begegnung mit inklusionsspezifischen Anforderungen bereits im Studium eine wichtige Rolle beigemessen werden. Eigenen, als bewältigbar erlebten Erfahrungen, etwa im Rahmen intensiv begleiteter Praktika (Müller 2010), kommt dabei eine wichtige Rolle zu.

Zusatzmaterial online Zusätzliche Informationen sind in der Online-Version dieses Artikels (https://doi. org/10.1007/s11618-021-01053-0) enthalten.

Förderung The study „Mehr Zeit für gute Schule“ („More Time for Good Schools“) was commissioned and funded by the Ministry of Culture of Lower Saxony. The Center for Applied Health Sciences (ZAG) at Leuphana University conducted the study.

Funding Open Access funding enabled and organized by Projekt DEAL.

Open Access Dieser Artikel wird unter der Creative Commons Namensnennung 4.0 International Lizenz veröffentlicht, welche die Nutzung, Vervielfältigung, Bearbeitung, Verbreitung und Wiedergabe in jeglichem Medium und Format erlaubt, sofern Sie den/die ursprünglichen Autor(en) und die Quelle ordnungsgemäß nennen, einen Link zur Creative Commons Lizenz beifügen und angeben, ob Änderungen vorgenommen wurden.

Die in diesem Artikel enthaltenen Bilder und sonstiges Drittmaterial unterliegen ebenfalls der genannten Creative Commons Lizenz, sofern sich aus der Abbildungslegende nichts anderes ergibt. Sofern das betreffende Material nicht unter der genannten Creative Commons Lizenz steht und die betreffende Handlung nicht nach gesetzlichen Vorschriften erlaubt ist, ist für die oben aufgeführten Weiterverwendungen des Materials die Einwilligung des jeweiligen Rechteinhabers einzuholen.

Weitere Details zur Lizenz entnehmen Sie bitte der Lizenzinformation auf http://creativecommons.org/ licenses/by/4.0/deed.de.

Interessenkonflikt M. Peperkorn, K. Müller und P. Paulus geben an, dass kein Interessenkonflikt besteht.

\section{Literatur}

Baeriswyl, S., Kunz Heim, D., \& Krause, A. (2017). Soziale Arbeitsbedingungen der Schweizer Lehrpersonen. Ergebnisse einer nationalen Studie. Schweizerische Zeitschrift für Bildungswissenschaften, $39(1), 95-112$.

Bakker, A.B., \& Demerouti, E. (2007). The job demands-resources model: state of the art. Journal of Managerial Psychology, 22(3), 309-328.

Bakker, A. B., \& Demerouti, E. (2017). Job demands-Resources theory: taking stock and looking forward. Journal of Occupational Health Psychology, 22(3), 273-285.

Bandura, A. (1997). Self-efficacy. The exercise of control. New York: Freeman.

Becker, P. (2006). Gesundheit durch Bedürfnisbefriedigung. Göttingen: Hogrefe.

Bennemann, E. M. (2019). Die Arbeits- und Gesundheitssituation von Lehrkräften: Aufgaben, Belastungen und Ressourcen an integrierten und inklusiven Schulen. Münster: Waxmann.

Bosse, S., Henke, T., Jäntsch, C., Lambrecht, J., Vock, M., \& Spörer, N. (2016). Die Entwicklung der Einstellung zum inklusiven Lernen und der Selbstwirksamkeit von Grundschullehrkräften. Empirische Sonderpädagogik, 1, 103-116. 
Bosse, S., Jäntsch, C., \& Spörer, N. (2015). Einschätzungen von Lehrerinnen und Lehrern zum inklusiven Unterricht. In N. Spörer, A. Schründer-Lenzen, M. Vock \& K. Maaz (Hrsg.), Inklusives Lernen und Lehren im Land Brandenburg. (Abschlussbericht zur Begleitforschung des Pilotprojekts ,Inklusive Grundschule“).

Bühner, M. (2011). Einführung in die Test- und Fragebogenkonstruktion (3. Aufl.). München: Pearson.

Cramer, C., Friedrich, A., \& Merk, S. (2018). Belastung und Beanspruchung im Lehrerinnen-und Lehrerberuf: Übersicht zu Theorien, Variablen und Ergebnissen in einem integrativen Rahmenmodell. bildungsforschung, 2018(1):1-23.

Dadaczynski, K., Paulus, P., \& Horstmann, D. (2019). The predictive value of individual and work-related resources for the health and work satisfaction of German school principals. Health Education Journal. https://doi.org/10.1177/0017896919867118.

Danner, D. (2015). Reliabilität die Genauigkeit einer Messung. Mannheim: GESIS Leibniz-Institut für Sozialwissenschaften. https://doi.org/10.15465/gesis-sg_011.

Demerouti, E., \& Nachreiner, F. (2019). Zum Arbeitsanforderungen-Arbeitsressourcen-Modell von Burnout und Arbeitsengagement - Stand der Forschung. Zeitschrift für Arbeitswissenschaft, 73(2), 119-130.

Döring-Seipel, E., \& Dauber, H. (2010). Was hält Lehrer und Lehrerinnen gesund - die Bedeutung von Ressourcen, subjektiver Bewertung und Verarbeitung von Belastung für die Lehrer und Lehrerinnen. Schulpädagogik heute, 1(2), 1-16.

Dumont, H. (2019). Neuer Schlauch für alten Wein? Eine konzeptuelle Betrachtung von individueller Förderung im Unterricht. Zeitschrift für Erziehungswissenschaft, 22(2), 249-277.

Ervasti, J., Kivimäki, M., Ichiro, K., Subramanian, S. V., Pentti, J., Ahola, K., Oksanen, T., Pohjonen, T., Vahtera, J., \& Virtanen, M. (2012). Pupils with special educational needs in basic education schools and teachers' sickness absences-A register-linkage study. Scandinavian Journal of Work and Environmental Health, 38, 209-217.

Gehrmann, A. (2013). Zufriedenheit trotz beruflicher Beanspruchungen? Anmerkungen zu den Befunden der Lehrerbelastungsforschung. In M. Rothland (Hrsg.), Belastung und Beanspruchung im Lehrerberuf (2. Aufl., S. 185-203). Wiesbaden: Springer.

Göransson, K., \& Nilholm, C. (2014). Conceptual diversities and empirical shortcomings-A critical analysis of research on inclusive education. European Journal of Special Needs Education, 29(3), 265-280.

Gräsel, C., Decristan, J., \& König, J. (2017). Adaptiver Umgang mit Heterogenität im Unterricht. Unterrichtswissenschaft, 45(4), 195-206.

Greiner, F., Taskinen, P., \& Kracke, B. (2020). Einstellungen und Selbstwirksamkeitsüberzeugungen von Lehramtsstudierenden bezüglich inklusiven Unterrichts: Zusammenhänge mit Kontakterfahrungen und Grundlagenkenntnissen über schulische Inklusion. Unterrichtswissenschaft, 48(2), $273-295$.

Grosche, M. (2015). Ein Diskussions- und Positionsartikel zur Definition von Inklusion aus Sicht der empirischen Bildungsforschung. In P. Kuhl, P. Stanat, B. Lütje-Klose, C. Gresch, H. A. Pant \& M. Prenzel (Hrsg.), Inklusion von Schülerinnen und Schülern mit sonderpädagogischem Förderbedarf in Schulleistungserhebungen (S. 17-39). Wiesbaden: Springer.

Hinds, E., Jones, L. B., Gau, J. M., Forrester, K. K., \& Biglan, A. (2015). Teacher distress and the role of experiential avoidance. Psychology in the Schools, 52(3), 284-297.

Hu, L.-T., \& Bentler, P. M. (1999). Cutoff criteria for fit indexes in covariance structure analysis: Conventional criteria versus new alternatives. Structural Equation Modeling, 6, 1-55.

Hulin, C.L., \& Judge, T. A. (2003). Job attitudes. In I. B. Weiner (Hrsg.), Handbook of psychology (S. 255-276). https://doi.org/10.1002/0471264385.wei1211.

Judge, T. A., \& Piccolo, R. (2004). Transformational and transactional leadership: A meta-analytic test of their relative validity. Journal of Applied Psychology, 89, 755-768.

Judge, T. A., Parker, S. K., Colbert, A. E., Heller, D. \& Ilies, R. (2002). Job satisfaction: A cross-cultural review. In N. Anderson, D. S. Ones, H. K. Sinangil \& C. Viswesvaran (Hrsg.), Handbook of industrial, work and organizational psychology. Volume 2: Organizational psychology (S. 25-52). Thousand Oaks, CA US: Sage Publications, Inc.

Kiel, E., Braun, A., Muckenthaler, M., Heimlich, U., \& Weiss, S. (2019). Self-efficacy of teachers in inclusive classes. European Journal of Special Needs Education. https://doi.org/10.1080/08856257. 2019.1683685 .

Kiel, E., Heimlich, U., Markowetz, R., Braun, A., \& Weiß, S. (2016). How to cope with stress in special needs education? Stress-inducing dysfunctional cognitions of teacher students: The perspective of professionalisation. European Journal of Special Needs Education, 31(2), 202-219. 
Klemm, K. (2018). Inklusion und Exklusion im deutschen Bildungssystem: Unterschiedliche Förderorte und schulische Leistungen. In K. Rathmann \& K. Hurrelmann (Hrsg.), Leistung und Wohlbefinden in der Schule: Herausforderung Inklusion (S. 33-45). Weinheim: Beltz Juventa.

Klusmann, U. (2011). Allgemeine berufliche Motivation und Selbstregulation. In M. Kunter, J. Baumert, W. Blum, U. Klusmann, S. Krauss \& M. Neubrand (Hrsg.), Professionelle Kompetenz von Lehrkräften: Ergebnisse des Forschungsprogramms COACTIV (S. 277-294). Münster: Waxmann.

Klusmann, U., \& Richter, D. (2014). Beanspruchungserleben von Lehrkräften und Schülerleistung: Eine Analyse des IQB-Ländervergleichs in der Primarstufe. Zeitschrift für Pädagogik, 60(2), 202-224.

König, J., Gerhard, K., Kaspar, K., \& Melzer, C. (2019). Professionelles Wissen von Lehrkräften zur Inklusion: Überlegungen zur Modellierung und Erfassung mithilfe standardisierter Testinstrumente. Pädagogische Rundschau, 73(1), 43-64.

Krause, A. (2004). Fragebogen zur Arbeitssituation an Schulen (FASS). Dokumentation der Skalen und Aussagen. Freiburg: Albert-Ludwigs-Universität.

Krause, A., \& Dorsemagen, C. (2014). Belastung und Beanspruchung im Lehrerberuf - Arbeitsplatz- und bedingungsbezogene Forschung. In E. Terhart, H. Bennewitz \& M. Rothland (Hrsg.), Handbuch der Forschung zum Lehrerberuf (S. 987-1013). Münster: Waxmann.

Kullmann, H., Lütje-Klose, B., \& Textor, A. (2014). Eine Allgemeine Didaktik für inklusive Lerngruppen fünf Leitprinzipien als Grundlage eines Bielefelder Ansatzes der inklusiven Didaktik. In B. Amrhein \& M. Dziak-Mahler (Hrsg.), Fachdidaktik inklusiv (S. 89-107). Münster: Waxmann.

Kunz Heim, D., Sandmeier, A., \& Krause, A. (2014). Negative Beanspruchungsfolgen bei Schweizer Lehrpersonen. Beiträge zur Lehrerinnen- und Lehrerbildung, 32(2), 280-295.

Kunz Heim, D., Sandmeier, A., Hänggi, Y., Safi, N., \& Cina, A. (2019). Training zum Umgang mit Unterrichtsstörungen: Effekte auf die Gesundheit von Lehrkräften. Zeitschrift für Erziehungswissenschaft, 22(4), 925-944.

Lütje-Klose, B., Neumann, P., Gorges, J., \& Wild, E. (2018). Die Bielefelder Längsschnittstudie zum Lernen in inklusiven und exklusiven Förderarrangements (BiLieF) - Zentrale Befunde. Die Deutsche Schule, 110(2), 9-23. https://doi.org/10.31244/dds.2018.01.02.

Maslach, C., Jackson, S. E., \& Leiter, M. P. (1997). Maslach burnout inventory: Third edition. In R. J. Wood \& C.P. Zalaquett (Hrsg.), Evaluating stress: A book of resources (S. 191-218). Lanham: The Scarecrow Press.

McElvany, N., Schroeder, S., Hachfeld, A., Baumert, J., Richter, T., Schnotz, W., Horz, H., \& Ullrich, M. (2009). Diagnostische Fähigkeiten von Lehrkräften: bei der Einschätzung von Schülerleistungen und Aufgabenschwierigkeiten bei Lernmedien mit instruktionalen Bildern. Zeitschrift für pädagogische Psychologie, 23(34), 223-235.

Melzer, C., Hillenbrand, C., Sprenger, D., \& Hennemann, T. (2015). Aufgaben von Lehrkräften in inklusiven Bildungssystemen - Review internationaler Studien. Erziehungswissenschaft, 26(51), 61-80.

Mohr, G., Rigotti, T., \& Müller, A. (2005). Irritation - ein Instrument zur Erfassung psychischer Beanspruchung im Arbeitskontext. Zeitschrift für Arbeits- u. Organisationspsychologie, 49(1), 44-48.

Müller, K. (2010). Das Praxisjahr in der Lehrerbildung - Empirische Befunde zur Wirksamkeit studienintegrierter Langzeitpraktika. Bad Heilbrunn: Klinkhardt.

Muthén, L. K., \& Muthén, B. O. (1998-2017). Mplus user's guide (8. Aufl.). Los Angeles: Authors.

Neumann, P., \& Lütje-Klose, B. (2020). Diagnostik in inklusiven Schulen - zwischen Stigmatisierung, Etikettierungs-Ressourcen-Dilemma und förderorientierter Handlungsplanung. In C. Gresch, P. Kuhl, M. Grosche, C. Sälzer \& P. Stanat (Hrsg.), Schüler*innen mit sonderpädagogischem Förderbedarf in Schulleistungserhebungen (S. 3-28). Wiesbaden: Springer VS.

Niedersächsisches Kultusministerium (2013). Verordnung zur Feststellung eines Bedarfs an sonderpädagogischer Unterstützung vom 22. Januar 2013. https://www.nds-voris.de/jportal/?quelle=jlink\& query $=\mathrm{SoP} \% \mathrm{C} 3 \% \mathrm{~A} 4 \mathrm{dFV}+\mathrm{ND}+\% \mathrm{C} 2 \% \mathrm{~A} 7+1 \& \mathrm{psml}=$ bsvorisprod.psml\&max=true. Zugegriffen: 1 . Juli 2020.

Niedersächsisches Kultusministerium (2018). Die niedersächsischen allgemein bildenden Schulen in Zahlen Stand: Schuljahr 2016/2017. https:/www.mk.niedersachsen.de/startseite/service/statistik/ allgemein_bildende_schulen/die-niedersaechsischen-allgemein-bildenden-schulen-in-zahlen- 6505 . html. Zugegriffen: 1. Juli 2020.

Nübling, M., Vomstein, M., Haug, A., Nübling, T., Stößel, U., Hasselhorn, H. M., \& Krause, A. (2012). Personenbezogene Gefährdungsbeurteilung an öffentlichen Schulen in Baden-Württemberg-Erhebung psychosozialer Faktoren bei der Arbeit. Freiburg: Freiburger Forschungsstelle Arbeits- und Sozialmedizin. 
Paulus, P., Horstmann, D., Baydar, C., \& Dadaczynski, K. (2017). Abschlussbericht zur Online-Befragung „Mehr Zeit für gute Schule“. https://www.leuphana.de/mehr-zeit-fuer-gute-schule/aktuell.html. Zugegriffen: 20. Juli 2020.

Paškvan, M., \& Kubicek, B. (2017). The intensification of work. In C. Korunka \& B. Kubicek (Hrsg.), Job demands in a changing world of work (S. 25-43). Cham: Springer.

Peperkorn, M., Müller, K., Mertz, K., \& Paulus, P. (2020). Dealing with inclusion-teachers' assessment of internal and external resources. International Journal of Inclusive Education. https://doi.org/10. $1080 / 13603116.2020 .1821450$.

Piwowar, V. (2013). Multidimensionale Erfassung von Kompetenzen im Klassenmanagement. Konstruktion und Validierung eines Beobachter- und eines Schülerfragebogens für die Sekundarstufe 1. Zeitschrift für pädagogische Psychologie, 27(4), 215-228.

Rathmann, K., \& Hurrelmann, K. (2018). Leistung und Wohlbefinden in der Schule: Herausforderung Inklusion. Weinheim: Beltz Juventa.

Rothland, M., \& Klusmann, U. (2016). Belastung und Beanspruchung im Lehrerberuf. In M. Rothland (Hrsg.), Beruf Lehrer/Lehrerin: Ein Studienbuch (S. 351-369). Stuttgart: UTB.

Rudow, B. (1994). Die Arbeit des Lehrers. Bern: Huber.

Sälzer, C., Gebhardt, M., Müller, K., \& Pauly, E. (2015). Der Prozess der Feststellung sonderpädagogischen Förderbedarfs in Deutschland. In P. Kuhl, P. Stanat, B. Lütje-Klose, C. Gresch, H. A. Pant \& M. Prenzel (Hrsg.), Inklusion von Schülerinnen und Schülern mit sonderpädagogischem Förderbedarf in Schulleistungserhebungen (S. 129-152). Wiesbaden: Springer VS.

Schmitz, G.S., \& Schwarzer, R. (2000). Selbstwirksamkeitserwartung von Lehrern: Längsschnittbefunde mit einem neuen Instrument. Zeitschrift für Pädagogische Psychologie, 14(1), 12-25.

Schult, J., Münzer-Schrobildgen, M., \& Sparfeldt, J.R. (2014). Belastet, aber hoch zufrieden? Arbeitsbelastung von Lehrkräften im Quer- und Längsschnitt. Zeitschrift für Gesundheitspsychologie, 22, 61-67.

Schwab, S. (2017). Interprofessionelle Lehrkraftkooperation im inklusiven Unterricht aus der Perspektive der Schülerinnen und Schüler. Unterrichtswissenschaft, 45(4), 262-279.

Schwarzer, R., \& Jerusalem, M. (Hrsg.) (1999). Skalen zur Erfassung von Lehrer- und Schülermerkmalen (Dokumentation der psychometrischen Verfahren im Rahmen der Wissenschaftlichen Begleitung des Modellversuchs Selbstwirksame Schulen). Berlin: Freie Universität.

Schwarzer, R., \& Warner, M. (2014). Forschung zur Selbstwirksamkeit bei Lehrerinnen und Lehrern. In E. Terhart, H. Bennewitz \& M. Rothland (Hrsg.), Handbuch der Forschung zum Lehrerberuf (2. Aufl., S. 662-678). Münster: Waxmann.

Tschannen-Moran, M., Hoy, A.W., \& Hoy, W. K. (1998). Teacher efficacy: Its meaning and measure. Review of educational research, 68(2), 202-248.

Turgut, S., Michel, A., \& Sonntag, K. (2014). Einflussfaktoren emotionaler Erschöpfung und Arbeitszufriedenheit. Zeitschrift für Arbeits- und Organisationspsychologie, 58(3), 140-154.

Urton, K. (2016). Faktoren des inklusiven Schulklimas als geteilte Merkmale von Schulleitungen, Kollegien und Lehrkräften (Dissertation). Universität Potsdam

Urton, K., Börnert-Ringleb, M., Krull, J., Wilbert, J., \& Hennemann, T. (2018). Inklusives Schulklima: Konzeptionelle Darstellung eines Rahmendmodells. Zeitschrift für Heilpädagogik, 69, 40-52.

Wang, M.-T., \& Degol, J.L. (2016). School climate: a review of the construct, measurement, and impact on student outcomes. Educational Psychology Review, 28(2), 315-352.

Weiber, R., \& Mühlhaus, D. (2010). Strukturgleichungsmodellierung. Berlin: Springer.

Weiss, H. M. (2002). Deconstructing job satisfaction: Separating evaluations, beliefs and affective experiences. Human Resource Management Review, 12, 173-194.

Weiß, S., Küchler, A., Muckenthaler, M., Heimlich, U., \& Kiel, E. (2019). „Die Belastungsgrenze ist überschritten?!“. Zeitschrift Für Inklusion, (3). https://www.inklusion-online.net/index.php/inklusiononline/article/view/498. Zugegriffen: 1. Juli 2020.

Werning, R., \& Arndt, A.-K. (Hrsg.). (2013). Inklusion: Kooperation und Unterricht entwickeln. Bad Heilbrunn: Klinkhardt. 